PACS: 12.20.-M; 31.15.AR; 31.25,-V; 31.30.JV; 32.10.FN

UDC 539.184

\title{
SENSING STRONG INTERACTION EFFECTS IN SPECTROSCOPY OF HADRONIC ATOMS
}

\author{
D. E. Sukharev', O. Yu. Khetselius', Yu. V. Dubrovskaya ${ }^{2}$ \\ ${ }^{1}$ I. I. Mechnikov Odessa National University, Odessa \\ ${ }^{2}$ Odessa State Environmental University, Odessa
}

\section{SENSING STRONG INTERACTION EFFECTS IN SPECTROSCOPY OF HADRONIC ATOMS}

\section{O. Yu. Khetselius, D. E. Sukharev, Yu. V. Dubrovskaya}

\begin{abstract}
The theoretical studying the strong interaction shifts and widths from X-ray spectroscopy of kaonic atoms is fulfilled. Sensing the strong interaction effects and theoretical estimating spectra of kaonic atomic systems can be considered as a new tool for studying nuclear structure and strong K-nucleus interaction.
\end{abstract}

Keywords: strong interaction effects, spectroscopy, kaonic atoms

\section{ДЕТЕКТУВАННЯ ЕФЕКТІВ СИЛЬНОЇ ВЗАЕМОДІЇ У СПЕКТРОСКОПІЇ АДРОННИХ АТОМІВ}

\section{О. Ю. Хецеліус, Д. Е. Сухарев, Ю. В. Дубровська}

Анотація. Виконано теоретичну оцінку зсувів і ширин рівнів, які обумовлені ефектами сильної взаємодії, в межах рентгенівської спектроскопії каонних атомів. Детектування ефектів сильної взаємодії і теоретична оцінка спектрів каонних атомів є одним з нових підходів до визначення ядерної структури і параметрів сильної каон- ядерної взаємодії.

Ключові слова: ефекти сильної взаємодії, спектроскопія, каонні атоми

\section{ДЕТЕКТИРОВАНИЕ ЭФФЕКТОВ СИЛЬНОГО ВЗАИМОДЕЙСТВИЯ В СПЕКТРОСКОПИИ АДРОННЫХ АТОМОВ}

\section{О. Ю. Хещелиус, Д. Е. Сухарев, Ю. В. Дубровская}

Аннотация. Выполнена теоретическая оценка сдвигов и ширин уровней, обусловленных эффектами сильного взаимодействия, в рамках рентгеновской спектроскопии каонных атомов. Детектирование эффектов сильного взаимодействия и оценка спектров каонных атомов являются одним из новых подходов к определению ядерной структуры и параметров сильного каон-ядерного взаимодействия.

Ключевые слова: эффекты сильного взаимодействия, спектроскопия, каонные атомы

\section{Introduction}

In last years studying the exotic hadronic atomic systems such as kaonic and pionic atoms are of a great interest for further development of atomic and nuclear theories as well as new tools for sensing the nuclear structure and fundamental kaon, pionnucleus strong interactions. Besides, studying these systems is very important for further check of the Standard model [1-16]. In the last few years transition energies in pionic [1] and kaonic atoms [2] have been measured with an unprecedented precision. The spectroscopy of kaonic hydrogen allows to study the strong interaction at low energies by measuring the energy and natural width of the 
ground level with a precision of few meV [1-5]. Besides, light kaonic atoms can additionally be used to dexne new low-energy X-ray standards [1] and to evaluate the kaon (pion) mass using high accuracy $\mathrm{X}$-ray spectroscopy. The collaborators of the E570 experiment $[6,7]$ measured X-ray energy of a kaonic helium atom, which is an atom consisting of a kaon (a negatively charged heavy particle) and a helium nucleus. Batty et al [4] had performed theoretical and experimental studying the strong-interaction effects in spectra of high $\mathrm{Z}$ kaonic atoms. These authirs had applied the nanve phenomenological optical model estimates. Now new exciting experiments are been preparing in order to make sensing the strong interaction effects in other hadronic atomic systems. The studies of the low-energy kaon-nuclear strong interaction with strangeness have been performed by measurements of the kaonic atom Xrays with atomic numbers $Z=1-92$ [1]. It is known that the shifts and widths due to the strong interaction can be systematically understood using phenomenological optical potential models. Nevertheless, one could mention a large discrepancy between the theories and experiments on the kaonic helium $2 p$ state. A large repulsive shift (about $-40 \mathrm{eV}$ ) has been measured by three experimental groups in the 1970's and 80's, while a very small shift $(<1 \mathrm{eV})$ was obtained by the optical models calculated from the kaonic atom $X$-ray data with $Z>2$ [1-6]. This significant disagreement (a difference of over 5 standard deviations) between the experimental results and the theoretical calculations is known as the "kaonic helium puzzle". A possible large shift has been predicted using the model assuming the existence of the deeply bound kaonic nuclear states. However, even using this model, the large shift of $40 \mathrm{eV}$ measured in the experiments cannot be explained. A re-measurement of the shift of the kaonic helium $\mathrm{X}$-rays is one of the top priorities in the experimental research activities. In the theory of the kaonic and pionic atoms there is an important task, connected with a direct calculation of the X-ray transition energies within consistent relativistic quantum mechanical atomic and nuclear theory methods. The standard way is based on solution of the KleinGordon equation, but there are many important problems connected with accurate accounting for as kaon-nuclear strong interaction effects as QED radiative corrections (firstly, the vacuum polarization effect etc.) [1-5]. This topic has been a subject of intensive theoretical and experimental interest (see [12-22]). In the present paper an effective ab initio approach to quantum Klein-Gordon equation calculation of X-ray spectra for multi-electron kaonic atoms with an account of the nuclear, radiative effects is proposed and the theoretical studying the strong interaction shifts and widths from X-ray spectroscopy of kaonic atoms is fulfilled. The level energies and energy shifts for these systems are estimated and in whole an analysis of the received data can be considered as a new tool for sensing the nuclear structure and strong kaon -nucleus interaction. The generalized optical potential model with correct defining the proton and neutron densities in a nucleus is used in direct definition of the strong interaction shifts and widths. It is carried out a detailed analysis of theoretical and experimental data on the strong interaction widths and shifts.

\section{New quantum Klein-Gordon equation approach in the kaonic atoms theory}

Let us describe the key moments of our new approach to quantum calculation of the spectra for multi-electron kaonic (pionic) atoms with an account of nuclear and radiative effects (more details applying to the multi-electron heavy atoms can be found in refs. [16-23]). It is well known that the relativistic dynamic of a spinless particle can be described by the Klein-Gordon equation. The electromagnetic interaction between a negatively charged spin-0 particle with a charge equal to $\mathrm{q}=-\mathrm{e}$ and the nucleus can be taken into account introducing the nuclear potential $\mathrm{A}_{v}$ in the $\mathrm{KG}$ equation via the minimal coupling $p_{v} \rightarrow p_{v}-q A_{v}$. The wave functions of the zeroth approximation for kaonic atoms are found from the Klein-Gordon equation [5]:

$$
m^{2} c^{2} \Psi(x)=\left\{\frac{1}{c^{2}}\left[i \hbar \partial_{t}+e V_{0}(r)\right]^{2}+\hbar^{2} \nabla^{2}\right\} \Psi(x),
$$

where $h$ is the Planck constant, c the velocity of the light and the scalar wavefunction $\Psi_{0}(\mathrm{x})$ depends on the space-time coordinate $\mathrm{x}=(\mathrm{ct}, \mathrm{r})$. Here it is considered a case of a central Coulomb potential $\left(\mathrm{V}_{0}(\mathrm{r})\right.$, 0 ). A usually, We consider here the stationary solution of Eq. (1). In this case, we can write:

$$
\Psi(\mathrm{x})=\exp (-\mathrm{iE} t / \hbar) \phi(x)
$$

and Eq. (1) becomes:

$$
\left\{\frac{1}{c^{2}}\left[E+e V_{0}(r)\right]^{2}+\hbar^{2} \nabla^{2}-m^{2} c^{2}\right\} \phi(x)=0
$$

where $\mathrm{E}$ is the total energy of the system (sum of the mass energy $\mathrm{mc}^{2}$ and binding energy $\varepsilon_{0}$ ). In prin- 
ciple, the central potential $V_{o}$ should include the central Coulomb potential, the vacuum-polarization potential as well as the kaon-nucleus strong interaction potential (optical model potential). Earlier we have calculated some characteristics of hydrogen-like and other multi-electron ions with using the nuclear charge distribution in the form of a uniformly charged sphere and Gaussian form (c.f. [19-21]). The advantage of the Gaussian form nuclear charge distribution is provided by using the smooth function instead of the discontinuous one as in the model of a uniformly charged sphere [22]. It is obvious that it simplifies the calculation procedure and permits to perform a flexible simulation of the real distribution of the charge in a nucleus. In last years to define the nuclear potential it is usually used the Fermi model for the charge distribution in the nucleus $\rho(r)$ (c.f.[21]):

$$
\left.c(r)=c_{0} /\{1+\exp [(r-c) / a)]\right\}
$$

where the parameter $\mathrm{a}=0.523 \mathrm{fm}$, the parameter $\mathrm{c}$ is chosen by such a way that it is true the following condition for average-squared radius: $\left\langle\mathrm{r}^{2}\right\rangle^{1 / 2}=$ $=\left(0.836 \cdot \mathrm{A}^{1 / 3}+0.5700\right) \mathrm{fm}$. Further let us present the formulas for the finite size nuclear potential and its derivatives on the nuclear radius. If the pointlike nucleus has the central potential W( R), then a transition to the finite size nuclear potential is realized by exchanging $\mathrm{W}(\mathrm{r})$ by the potential [19]:

$$
\begin{gathered}
W(r \mid R)=W(r) \int_{0}^{r} d r r^{2} \rho(r \mid R)+ \\
+\int_{r}^{\infty} d r r^{2} W(r) \rho(r \mid R) .
\end{gathered}
$$

We assume it as some zeroth approximation. Further the derivatives of various characteristics on $\mathrm{R}$ are calculated. They describe the interaction of the nucleus with outer electron; this permits recalculation of results, when $\mathrm{R}$ varies within reasonable limits. The Coulomb potential for the spherically symmetric density $\rho(r \mid R)$ is:

$$
\begin{aligned}
V_{\text {nucl }}(r \mid R) & =-\left((1 / r) \int_{0}^{r} d r^{\prime} r^{\prime 2} \rho\left(r^{\prime} \mid R\right)+\right. \\
& +\int_{r}^{\infty} d r^{\prime} r^{\prime} \rho\left(r^{\prime} \mid R\right)
\end{aligned}
$$

It is determined by the following system of differential equations [19]:

$$
V^{\prime} n u c l(r, R)=\left(1 / r^{2}\right) \int_{0}^{r} d r^{\prime} r^{\prime 2} \rho\left(r^{\prime}, R\right) \equiv\left(1 / r^{2}\right) y(r, R)
$$

$$
\begin{gathered}
y^{\prime}(r, R)=r^{2} \rho(r, R) \\
\left.c^{\prime}(r)=\left(c_{0} / a\right) \exp [(r-c) / a]\{1+\exp [(r-c) / a)]\right\}^{2}
\end{gathered}
$$

with the boundary conditions:

$$
\begin{gathered}
V_{\text {nucl }}(0, R)=-4 /(\pi r) \\
y(0, R)=0,(8) \\
c(0)=c_{0} /\{1+\exp [-c / a]\}
\end{gathered}
$$

The new important topic is connected with a correct accounting the radiation QED corrections and, first of all, the vacuum polarization correction. Procedure for an account of the radiative QED corrections in a theory of the multi-electron atoms is given in detail in refs. [17-22]. Regarding the vacuum polarization effect let us note that this effect is usually taken into account in the first PT order by means of the Uehling potential:

$$
\begin{aligned}
& U(r)=-\frac{2 \alpha}{3 \pi r} \int_{1}^{\infty} d t \exp (-2 r t / \alpha Z) \times \\
& \times\left(1+1 / 2 t^{2}\right) \frac{\sqrt{t^{2}-1}}{t^{2}} \equiv-\frac{2 \alpha}{3 \pi r} C(g),
\end{aligned}
$$

where $g=\frac{r}{\alpha Z}$. In our calculation we usually use more exact approach. The Uehling potential, determined as a quadrature (9), may be approximated with high precision by a simple analytical function. The use of new approximation of the Uehling potential [21] permits one to decrease the calculation errors for this term down to $0.5-1 \%$. Besides, using such a simple analytical function form for approximating the Uehling potential allows its easy inclusion into the general system of differential equations.

As it is well known, the nuclear absorption is defined by the strength of the strong interaction and overlapping the kaonic atomic wave function with the nuclear ones. The widespread approach to treating the strong interaction between the nucleus and orbiting kaon is in using the phenomenological optical potential of the following form $[1,5,10]$ :

$$
V_{N}=-\frac{2 \pi}{\mu}\left[1+\frac{M_{K}}{M_{N}}\right]\left[A_{K p} \rho_{p}(r)+A_{K n} \rho_{n}(r)\right],
$$

where $\mu$ is the kaon-nucleus reduced mass, $M_{K}$ and $M_{N}$ are the kaon and nucleon masses, $\rho_{p}(r), \rho_{n}(r)$ are the proton and neutron densities in the nucleus and $A_{K p}, A_{K n}$ are the corresponding complex effective $K p$ and $K n$ scattering lengths. It si well known the Batty simplifying assumption of the following kind [4]: 


$$
V_{N}=-\frac{2 \pi}{\mu}\left[1+\frac{M_{K}}{M_{N}}\right][a \rho(r)],
$$

where $a$ is the effective averaged K-nucleon scattering length. Batty et al had analyzed the previous kaon data and found the acceptable value for the a length is as follows [4]:

$$
a=[(0.34 \pm 0.03)+\mathrm{i}(0.84 \pm 0.03)](\mathrm{fm}) .
$$

The presented value of the length a has been indeed chosen to describe the low and middle $\mathrm{Z}$ nuclei [4]. The disadvantage of the usually used approach is connected with approximate definition of the proton and neutron densities and using the effective averaged K-nucleon scattering length. More correct approach is in the the relativistic mean-field (RMF) model for the ground-state calculation of the nucleus. Though we have no guaranty that these wave-functions yield a close approximation to nature, the success of the RMF approach supports our choice [24]. These wave functions do not suffer from known deficiencies of other approaches, e.g., the wrong asymptotics of wave functions obtained in a harmonic oscillator potential. The RMF model has been designed as a renormalizable meson-field theory for nuclear matter and finite nuclei [24]. The realization of nonlinear self-interactions of the scalar meson led to a quantitative description of nuclear ground states. As a self-consistent mean-field model (for a comprehensive review see ref. [22-24]), its ansatz is a Lagrangian or Hamiltonian that incorporates the effective, in-medium nucleon-nucleon interaction. Recently [22] the self-consistent models have undergone a reinterpretation, which explains their quantitative success in view of the facts that nucleons are composite objects and that the mesons employed in RMF have only a loose correspondence to the physical meson spectrum. They are seen as covariant Kohn-Sham schemes and as approximations to the true functional of the nuclear ground state. As a Kohn-Sham scheme, the RMF model can incorporate certain ground-state correlations and yields a ground-state description beyond the literal mean-field picture. RMF models are effective field theories for nuclei below an energy scale of $1 \mathrm{GeV}$, separating the long- and intermediate-range nuclear physics from short-distance physics, involving, i.e., short-range correlations, nucleon form factors, vacuum polarization etc, which is absorbed into the various terms and coupling constants. As it is indicated in refs. [24] the strong attractive scalar $(S:-400 \mathrm{MeV})$ and repulsive vector $(V:+350 \mathrm{MeV})$ fields provide both the binding mechanism $(S+V$ : $-50 \mathrm{MeV})$ and the strong spin-orbit force $(S-V:-$ $750 \mathrm{MeV}$ ) of both right sign and magnitude. In our calculation we have used so called NL3-NLC (see details in refs. [24]), which is among the most successful parameterizations available.

\section{Results and conclusions}

In ref. [5] we have presented some calculations for a selection of kaonic atom transitions. Such calculations are obtained solving numerically the Klein-Gordon equation using the effective Dirac Superatom code developed by Ivanov et al [16-21] that has been modified to include spin- 0 particles case, even in the presence of electrons [1]. The kaon mass was assumed to be $493.677 \pm 0.013 \mathrm{MeV}$ [11]. In table 1 we present the calculated electromagnetic (EM) X-ray energies of kaonic atoms for transitions between circular levels. The transitions are identified by the initial $\left(n_{i}\right)$ and final $\left(n_{f}\right)$ quantum numbers. The calculated values of transition energies are compared with available measured $\left(\mathrm{E}_{\mathrm{m}}\right)$ and other calculated $\left(E_{c}\right)$ values [1-7]. In a case of the close agreement between theoretical and experimental data, the corresponding levels are less sensitive to strong nuclear interaction. In the opposite case one could point to a strong-interaction effect in the exception cited above.

Calculated $\left(\mathrm{E}_{\mathrm{c}}\right)$ and measured $\left(\mathrm{E}_{\mathrm{m}}\right)$ kaonic atoms $\mathrm{X}$-ray energies (in keV)

Table 1

\begin{tabular}{|c|c|c|c|c|c|c|}
\hline Nucl. & Transition & $\mathrm{E}_{\mathrm{c}}$, our theor & $\mathrm{E}_{\mathrm{c}},[4]$ & $\mathrm{E}_{\mathrm{c}}[6]$ & $\mathrm{E}_{\mathrm{c}}[7]$ & $\mathrm{E}_{\mathrm{m}}$ \\
\hline $\mathrm{W}$ & $8-7$ & 346.572 & 346.54 & - & - & $346.624(25)$ \\
\hline $\mathrm{W}$ & $7-6$ & 535.136 & 535.24 & - & - & $534.886(92)$ \\
\hline $\mathrm{Pb}$ & $8-7$ & 426.174 & 426.15 & - & - & $426.221(57)$ \\
\hline $\mathrm{U}$ & $8-7$ & 538.528 & 538.72 & 538.013 & 537.44 & $538.315(100)$ \\
\hline
\end{tabular}

In table 2 we present the calculated ( $C$ ) and measured (M) strong interaction shifts $\Delta \mathrm{E}$ and widths $\mathrm{G}$ (in $\mathrm{keV}$ ) for the kaonic atoms $\mathrm{X}$-ray transitions. The subscripts $\mathrm{M}$ and $\mathrm{C}$ stands for measured and calculated values correspondingly. The width $\mathrm{G}$ is the strong width of the lower level which was obtained by 
subtracting the electromagnetic widths of the upper and lower level from the measured value. The shift $\Delta \mathrm{E}$ is defined as difference between the measured $E_{M}$ and calculated $E_{E M}$ (electromagnetic) values of transition energies; the calculated value is obtained by direct solving the equation (1) with kaon-nuclear potential. Besides, the measured values by Miller et al and Cheng et al (from refs. [1,4] are listed in table 2 too. It should be noted that Cheng et al did not make any energy calibration above the $511 \mathrm{e}^{+}$annihilation and Batty et al [4] indicated that the corresponding difference between the energy values is not serious.

Table 2

Calculated ( $\mathrm{C}$ ) and measured ( $\mathrm{M}$ ) strong interaction shifts $\Delta \mathrm{E}$ and widths $\mathrm{G}$ for the kaonic atoms X-ray transitions: a- the shift was estimated with Miller et al measured energy (see [1]); b - the shift was estimated with Cheng et al measured energy (see [1]); c - the shift by Batty et al [4]; $\mathrm{d}$ - this work;

\begin{tabular}{|c|c|c|c|c|c|c|}
\hline Nucl & $\Delta \mathrm{E}_{\mathrm{C}}(\mathrm{d})$ & $\mathrm{G}_{\mathrm{C}}(\mathrm{d})$ & $\Delta \mathrm{E}_{\mathrm{C}}(\mathrm{c})$ & $\mathrm{G}_{\mathrm{C}}(\mathrm{c})$ & $\Delta \mathrm{E}_{\mathrm{M}}$ & $\mathrm{G}_{\mathrm{M}}$ \\
\hline $\mathrm{W}, 8-7$ & 0.038 & 0.072 & -0.003 & 0.065 & $\begin{array}{l}0.079^{\mathrm{c}} \\
0.052^{\mathrm{d}}\end{array}$ & $0.070(15)$ \\
\hline $\mathrm{W}, 7-6$ & -0.294 & 3.85 & -0.967 & 4.187 & $\begin{array}{l}-0.353^{\mathrm{c}} \\
-0.250^{\mathrm{d}}\end{array}$ & $3.72(35)$ \\
\hline $\mathrm{Pb}, 8-7$ & 0.035 & 0.281 & -0.023 & 0.271 & $\begin{array}{l}0.072^{\mathrm{c}} \\
0.047^{\mathrm{d}}\end{array}$ & $\begin{array}{c}0.284(14) \\
0.370(150)^{\mathrm{a}}\end{array}$ \\
\hline $\mathrm{U}, 8-7$ & -0.205 & 2.620 & -0.189 & 2.531 & $\begin{array}{c}0.120^{\mathrm{a}} \\
0.032^{\mathrm{b}} \\
-0.40^{\mathrm{c}} \\
-0.213^{\mathrm{d}}\end{array}$ & $\begin{array}{c}2.67(10) \\
1.50(75)^{\mathrm{a}}\end{array}$ \\
\hline
\end{tabular}

From the other side, more correct definition of proton and neutron densities is of a great importance for physically reasonable agreement between the measured and calculated (this work) shifts and widths. In whole we can conclude that the measured strong interaction parameters are reasonably well reproduced by present theory. To understand further information on the low-energy kaon-nuclear interaction, new experiments to determine the shift and width of kaonic atoms are now in preparation in J-Parc and in LNF, respectively (look, for example, refs. $[1,8])$.

\section{References}

1. Deloff A., Fundamentals in Hadronic Atom Theory, Singapore: World Scientific, 2003. - 352P.

2. Hayano R. S., Hori M., Horvath D., Widman E., Antiprotonic helium and CPT invariance//Rep. Prog. Phys. - 2007. - Vol. 70. - P. 1995-2065.

3. Deslattes R., Kessler E., Indelicato P., de Billy L., Lindroth E., Anton J., Exotic atoms//Rev. Mod. Phys. - 2003. - Vol. 75. - P. 35-70.

4. Batty C. J., Eckhause M., Gall K. P., et al. Strong interaction effects in high $\mathrm{Z}-\mathrm{K}^{-}$atoms//Phys. Rev. C. - 1989. - Vol. 40. - P. 2154-2160.

5. Khetselius O. Yu., Turin A. V., Sukharev D. E., Florko T. A., Estimating of X-ray spectra for kaonic atoms as tool for sensing the nuclear structure// Sensor Electr. and Microsyst. Techn. - 2009. - N1. P. 30-35.
6. Okada S., Beer G., Bhang H., et al, Precision measurement of the $3 \mathrm{~d} \rightarrow 2 \mathrm{p}$ x-ray energy in kaonic ${ }^{4} \mathrm{He} / /$ Phys. Lett. B. - 2007. - Vol. 653, N 5-6. - P. 387391.

7. Ito T. M., Hayano R. S., Nakamura S. N., Terada T. P., Observation of kaonic hydrogen atom x rays// Phys. Rev. C. - 1998. - Vol. 58. - P. 2366 - 2382

8. Ishiwatari T. on behalf of the SIDDHARTA Collaboration, Silicon drift detectors for the kaonic atom Xray measurements in the SIDDHARTA experiment// Nucl. Instr. and Methods in Physics. Research Sec. A. Accelerators, Spectrometers, Detectors and Associated Equipment. - 2007. - Vol. 581,N1-2. P. 326-329.

9. Glushkov A. V., Makarov I. T., Nikiforova E. S., Pravdin M. I., Sleptsov I. Ye., Muon component of EAS with energies above $10^{17} \mathrm{eV} / /$ Astropart. Phys. 1995. - Vol. 4. - P. 15-22

10. Glushkov A. V., Khetselius O. Yu., Gurnitskaya E. P., Loboda A. V., Sukharev D. E., Relativistic Quantum Chemistry of Heavy Ions and Hadronic Atomic Systems: Spectra and Energy Shifts// Theory and Applications of Computational Chemistry (AIP). 2009. - Vol. 1. - P. 131-134.

11. Santos J. P., Parente F., Boucard S., Indelicato P., Desclaux j. P., X-ray energies of circular transitions and electron scattering in kaonic atoms//Phys. Rev. A. - 2005. - Vol. 71. - P. 032501.

12. Mohr P. J. Quantum Electrodynamics Calculations in few-Electron Systems// Phys. Scripta. - 1993. Vol. 46,N1. - P. 44-52.

13. Grant I., Relativistic Quantum Theory of Atoms and Molecules Theory and Computation, Springer Series 
on Atomic, Optical, and Plasma Physics. 2007. Vol. 40. - P. 587-626.

14. Quiney H. M., Grant I. P. Partial-wave mass renormalization in atomic QED calculation // Phys. Scripta T. - 1993. - Vol. 46. - P. 132-138.

15. Wilson S., Recent Advances in Theoretical Physics and Chemistry Systems//Recent Advances in Theor. Phys. and Chem. Systems (Springer). - 2006. Vol. 15. - P. 11-45.

16. Ivanova E. P., Ivanov L. N., Glushkov A. V., Kramida A. E. High order corrections in the Relativistic Perturbation Theory with the model Zeroth Approximation, Mg-like and Ne-like ions //Phys. Scripta 1985. - Vol. 32,N4. - P. 512-524.

17. Glushkov A. V., Energy Approach to Resonance states of compound super-heavy nucleus and EPPP in heavy nucleus collisions// Low Energy Antiproton Phys. (AIP). - 2005. - Vol. 796. - P. 206-210.

18. Glushkov A. V., Rusov V. D., Ambrosov S. V., Loboda A. V., Resonance states of compound super-heavy nucleus and EPPP in heavy nucleus collisions // New Projects and New Lines of research in Nuclear physics. Eds. Fazio G. and Hanappe F.: Singapore, World Sci. - 2003. - P. 142-154.

19. Glushkov A. V., Relativistic quantum theory. Quantum mechanics of atomic systems, Odessa: Astroprint, 2008. - 900P.

20. Glushkov A. V., Ambrosov S. V., Khetselius O. Yu.,
Loboda A. V., Gurnitskaya E. P., QED calculation of heavy multicharged ions with account for the correlation, radiative and nuclear effects// Recent Advances in Theory of Phys. and Chem. Systems (Springer). 2006. - Vol. 15. - P. 285-300.

21. Glushkov A. V., Khetselius O. Yu., Lovett L., et al, Gauge-invariant QED perturbation theory approach to calculating nuclear electric quadrupole moments, hyperfine structure constants for heavy atoms and ions// Frontiers in Quantum Systems in Chemistry and Physics (Springer). - 2008. - Vol. 18. - P. 505522.

22. Glushkov A. V., Lovett L., Khetselius O. Yu., Gurnitskaya E. P., Dubrovskaya Yu. V., Loboda A. V., Generalized multiconfiguration model of decay of multipole giant resonances applied to analysis of reaction $(\mu-n)$ on the nucleus ${ }^{40} \mathrm{Ca} / /$ International Journal of Modern Physics A. Particles and Fields. 2009. - Vol. 24. - P. 611-615.

23. Glushkov A. V., Malinovskaya S. V., Dubrovskaya Yu. V., Vitavetskaya L. A., Quantum calculation of cooperative muon-nuclear processes: discharge of metastable nuclei during negative muon capture// Recent Advances in Theory of Phys. and Chem. Systems (Springer). - 2006. - Vol. 15. - P. 301-318.

24. Serot B. D., Walecka J. D., Advances in Nuclear Physics Vol. 16: The Relativistic Nuclear Many Body Problem. Plenum Press, New York, 1986. 